\title{
Design and Simulation of Sensor Based on One Dimensional Photonic Crystal Metallodielectric with Two Defect
}

\author{
Teguh Puja Negara, Agung Prajuhana
}

\begin{abstract}
In this paper we report characteristics of a $1 D$ metallodielectric photonic crystal with a two defect rod. Simulations of the field propagation for the corresponding Transverse Electric (TE) modes were carried out using Finite Difference Time Domain (FDTD) technique on Maxwell's Equation. The results show that for certain chosen parameters of the transmittance varies if the refractive index of the defect rod is changed and that approximate linier changes for an refractive index range of 1.3 - 1.5 with a slight increase for a second defect change and a relatively steep decrease for a first defect change. A second defect index change can be used as the first defect sensitivity control The characteristics change due to variation of the defect rod radius is also considered for sensor design optimization
\end{abstract}

Index Terms: Photonic Crystal, Metalodielectric, Optical Sensor, FDTD Method

\section{INTRODUCTION}

The propagation of electromagnetic waves in various types of photonics crystals (PCs) has been intensely investigated by researchers due to its interesting applications in optics since their discoveries by E. Yablonovitch and S. John [1] independently. The optical sensor model described in this paper consists of a one-dimensional (1D) photonic crystal (PhC) metallodielectric by two defects. Numerical simulations were performed by applying the Finite Difference Time Domain (FDTD) method [2]. It is well known for TE polarizations that the coupling between the free electrons in the metal and the electric wave generates surface plasmons [3]. The existence of surface plasmons in metallic layered structure has been widely studied, such as in the binary metallic grating with a small gap used as optical filter or biosensor [4] or metal-dielectric-metal (MDM) as cavity or waveguide in photonic devices [5].

A sensor will work if there is a strong interaction between the sensor and sample material. However previous research on a $1 \mathrm{D} \mathrm{PhC}$ optical sensor for index measurement is that it will only work for a specific sample material within a certain measurable range [6]. In this paper we describe results from our numerical studies on a $1 \mathrm{D} \mathrm{PhCmetallodielectric} \mathrm{with} \mathrm{two}$ defect that respon the time average energy density $\mathrm{W}$ linear to change of refractive index of material of first defect and second defect. The second defect response is more linear than the first defect so that the second defect can be used as a receptor for the sensor while the first defect can be used as a control to regulate sensitivity. The rod diameter size variation is also taken into account to see the maximum time average energy density

\section{FDTD METHOD}

Finite Difference Time Domain (FDTD) is a method that uses an approach to the domain of space and time. The FDTD method was first introduced by Kane Yee in 1966 to analyze the electromagnetic field. This method is now widely applied as a major method of analysis. For two-dimensional electromagnetic wave with TE (transverse electric) mode can be written [7]

$$
\begin{aligned}
H_{z}^{n+\frac{1}{2}}\left(i+\frac{1}{2}, j+\frac{1}{2}\right)= & H_{z}^{n+\frac{1}{2}}\left(i+\frac{1}{2}, j+\frac{1}{2}\right)-\frac{\Delta t}{\mu\left(i+\frac{1}{2}, j+\frac{1}{2}\right)} \\
& \left(\begin{array}{c}
\frac{1}{\Delta x}\left(E_{y}^{n}\left(i+\frac{1}{2}, j+\frac{1}{2}\right)-E_{y}^{n}\left(i, j+\frac{1}{2}\right)\right)- \\
\frac{1}{\Delta y}\left(H_{x}^{n}\left(i+\frac{1}{2}, j+1\right)-H_{x}^{n}\left(i+\frac{1}{2}, j\right)\right)
\end{array}\right) \\
E_{z}^{n}\left(i+\frac{1}{2}, j\right)= & \frac{\varepsilon\left(i+\frac{1}{2}, j\right)}{\varepsilon\left(i+\frac{1}{2}, j\right)+\sigma\left(i+\frac{1}{2}, j\right) \Delta t} E_{z}^{n-1}\left(i+\frac{1}{2}, j\right)- \\
& \frac{\Delta t}{\varepsilon\left(i+\frac{1}{2}, j\right)+\sigma\left(i+\frac{1}{2}, j\right) \Delta t} \frac{1}{\Delta y} \\
& \frac{\left(\frac{1}{\Delta x}\left(H_{z}^{n-\frac{1}{2}}\left(i+\frac{1}{2}, j+\frac{1}{2}\right)-H_{z}^{n-\frac{1}{2}}\left(i-\frac{1}{2}, j+\frac{1}{2}\right)\right)\right)}{\varepsilon\left(i, j+\frac{1}{2}\right)+\sigma\left(i, j+\frac{1}{2}\right) \Delta t} \frac{1}{\Delta x} \\
E_{y}^{n}\left(i, j+\frac{1}{2}\right)= & \frac{\varepsilon\left(i, j+\frac{1}{2}\right)+\sigma\left(i, j+\frac{1}{2}\right) \Delta t}{\left(\frac{1}{\Delta x}\left(H_{z}^{n-\frac{1}{2}}\left(i+\frac{1}{2}, j+\frac{1}{2}\right)-H_{z}^{n-\frac{1}{2}}\left(i-\frac{1}{2}, j+\frac{1}{2}\right)\right)\right)} \\
& \frac{\Delta t}{\varepsilon-1}\left(i, j+\frac{1}{2}\right)-
\end{aligned}
$$

Revised Manuscript Received on April 25, 2019.

Teguh Puja Negara, Department of Physics, Bogor Agricultural University, Bogor 16680, Indonesia

Agung Prajuhana, Department of Computer Science, Pakuan University, Bogor 16143, Indonesia. 


\section{Design and Simulation of Sensor Based on One Dimensional Photonic Crystal Metallodielectric with Two Defect}

To investigate the change in the electric field due to material changes in the defect rod, we consider the amount of energy at the end of the device as a function of time, defined by:

$$
Q_{t}(t)=\int_{0}^{h} \varepsilon|\vec{E}(t)|^{2} d y
$$

with $\vec{E}$ denoting the TE case of the electric field at the left end of the slab and $h$ is the slab thickness. Further, we define the following parameter for a given refractive index of defect rod

$$
W=\frac{1}{t} \int_{0}^{t} Q(t) d t
$$

which describes the time average energy density, where $t$ is the corresponding measurement time.

The steady state, continuous wave $(\mathrm{CW})$ field $E(\omega)$ is calculated from $E(t)$ by Fourier transform during the simulation [8]

$E(\omega)=\int_{0}^{t} e^{i \omega t} E(t) d t$

Then the stored data is Fourier transformed and integrated. Finally, the ratio is taken between obtained integrated results to incident spectra which results in transmission spectra versus wavelength. The transmittance of structure is

$T=\left|\frac{E_{\text {trans }}(\omega)}{E_{\text {inc }}(\omega)}\right|^{2}$

Model structure consisting of eleven alternating dielectric and metallic rods, with the eleven dielectric rods and two rods is considered as a defect.

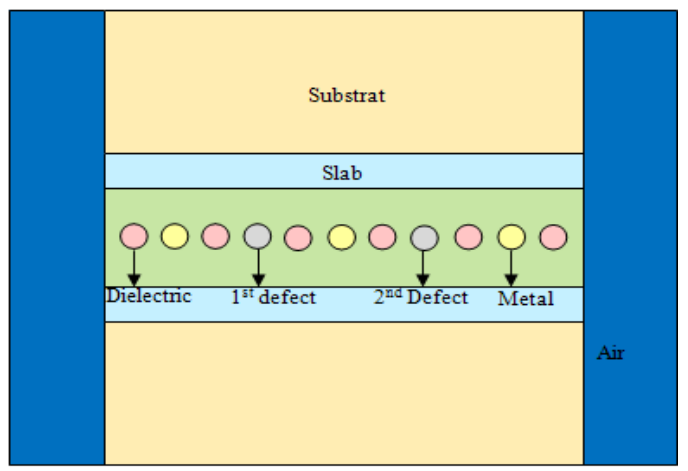

Fig. 1:One Dimensional Photonic Crystal Structure

The sketch of the model is shown in Fig. 1 which is homogeneous in the $z$-direction. It is important to note that because the periodicity of the system is only in one dimension, the model is called 1D PhC. For the numerical simulation we use eleven periodic rods for the dielectric material namely silicon ( $\mathrm{Si}$ ) with relative permittivity of $\varepsilon_{r}=12.11$ and relative permeability of $\mu_{r}=1$. For the metallic rod, we choose silver $(\mathrm{Ag})$ with a relative permittivity $\varepsilon_{r}=18.36+4.38 i$ at $700 \mathrm{~nm}$ based on the
Brendel-Borman model [9], and a relative permeability $\mu_{r}=0.99998$.

The length of device is $L=240 \mu m$ and thickness is $h=30 \mu \mathrm{m}$. We consider the refractive indices of the slab and substrate to be 1.44 and 2.21 respectively, with the corresponding thickness is $h=30 \mu \mathrm{m}$

\section{RESULTS AND DISCUSSION}

In our simulation we use a mesh number of $300 \times 300$. Each mesh has a size of $\Delta x=\Delta y=1 \mu m$, while the time step is taken to be $\Delta t=20 \mathrm{~ns}$. For the change of refractive index on second defect with range 1.3-1.5 obtained the transmittance response is more linear than at the change of refractive index at first defect. The sensitivity of the first defect was found 1.2918 while the sensitivity of the second defect is 1.6271 . Based on these results, the first defect can be used as a receptor on the optical sensor by selecting the sensing index $1.3-1.5$

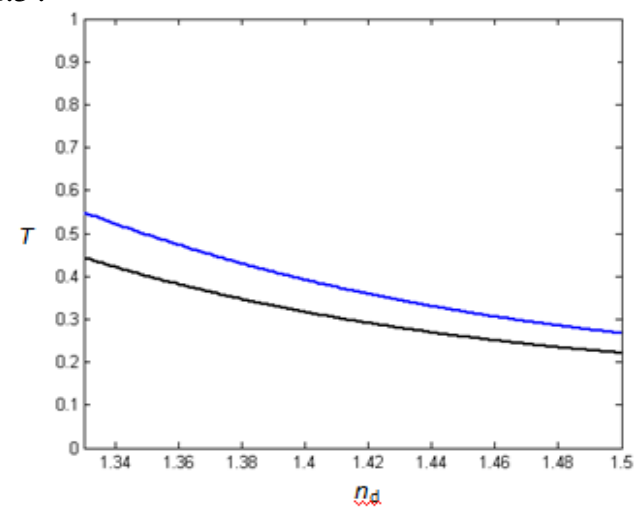

Fig. 2: The transmittance of second defect for first defect $\left(\mathrm{n}_{\mathrm{d} 1}\right)$ is 2.1 (blue). The transmittance of firs defect for second defect $\left(\mathrm{n}_{\mathrm{d} 2}\right)$ is 2.1 (black)

The second defect sensitivity value can be adjusted by varying the refractive index value of the first defect. Figure 3 shows the second defect sensitivity value that changed by 1.6271 for the refractive index of first defect of $2.1,1.0916$ for the refractive index of first defect of 2.0, and 0.68951 for the refractive index of first defect of 1.9. With this result, the first defect can be used as a sensitivity control for the sensor device.

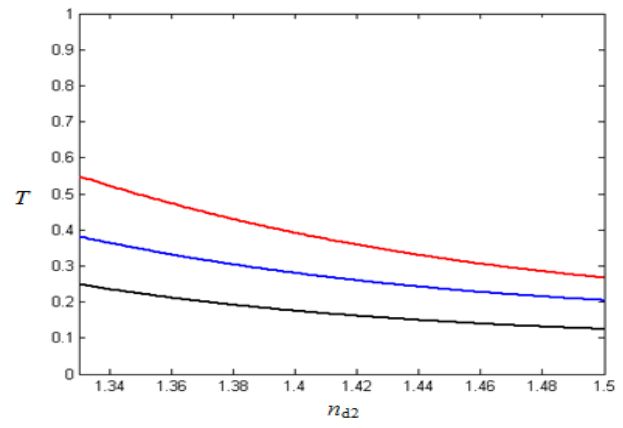

Fig. 3:Plot of transmittance to the variation of refractive index of the second defect 2.1 (red), 2.0 (blue), and 1.9

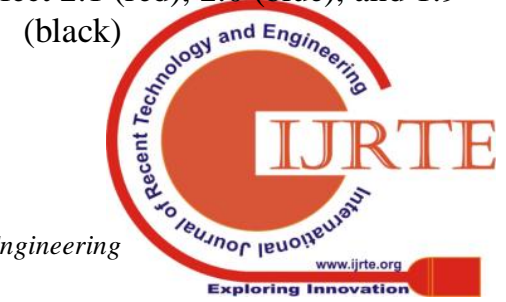


The other feature that was simulated is the effect of changing the refractive index of the second defect for a changing the rod radius and measure the energy density at the output. Based on figure 4 , it is seen that the radius value of the circle affects the energy density value. for the range of refractive index on the second defect, the value of $7 \mathrm{~mm}$ radius is very influential on the change in energy density, so that the radius value can be used for the sensor device

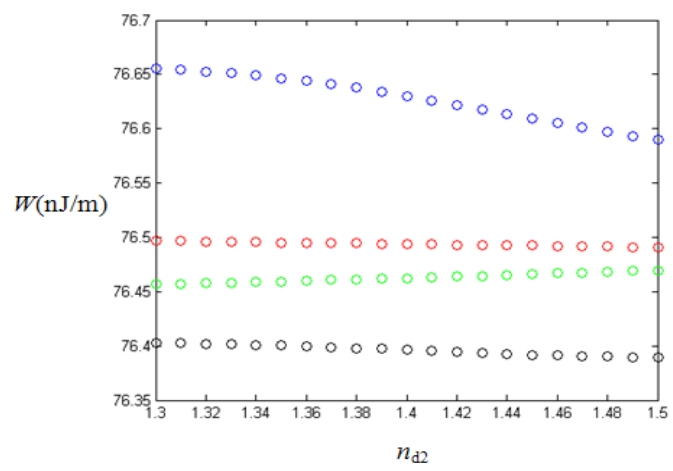

Fig. 4:The change of refractive index respect to energy density with a variety of radius: $r e d(r=4 \mathrm{~mm})$, green $(\mathrm{r}=$

$5 \mathrm{~mm})$, black $(\mathrm{r}=6 \mathrm{~mm})$, blue $(\mathrm{r}=7 \mathrm{~mm})$

\section{CONCLUSION}

Based on the results of this study, we get a model of photonic crystal structure which can be used as a sensor device. The first defect of photonic crystal can be used for sensing material while the second defect of photonic crystal can be used for sensitivity control. the size of the diameter of the defect hole can be considered to obtain optimal results.

\section{ACKNOWLEDGEMENT}

Teguh Puja Negara would like to thank Faculty of Mathematics and Natural Sciences, Pakuan University, for financial support.

\section{REFERENCES}

1. $\quad$ E. Yablonovitch. "Inhibited spontaneous emission in solid-state physics and electronics.” Physical review letters, vol. 58, no. 20, art. no. 2059, 1987.

2. A. Taflove and S. C. Hagness, Computational Electrodynamics: Finite-Different Time-Domain Method. London: Artech House, 2000.

3. G. Acuna, S. F. Heucke, F. Kuchler, H. T. Chen, A. J. Taylor and R. Kersting. "Surface Plasmons inTerahertz Metamaterial." Optics Express. vol. 16, pp. 18745-18751, 2008.

4. V. M. Fitio, H. P. Laba, and V. V. Bobotski, "Electromagnetic resonance absorption in metallic gratings." Semiconductor Physics, Quantum Electronics \& Optoelectronic, vol. 9, no. 3, pp. 49-55, 2006.

5. Z. Han, and S. He, "Multimode interference effect in plasmonic sub-wavelength waveguides and an ultra-compact power splitter," Optics communications. vol. 278, no. 1, pp. 199-203, 2007.

6. H. Alatas, H. Mayditia, A. A. Iskandar and M. O. Tjia, "Single Frequency refractive Index Sensor Based on a Finite One Dimensional Photonic Crystals With Two Defects," Japanese journal of applied physics, vol. 45, no. 8S, pp. 6754-6758, 2006.

7. K. Yee, "Numerical Solution of Initial Boundary Value Problems Involving Maxwell's Equation in Isotropic Media," IEEE Transactions on antennas and propagation, vol. 14, no. 3, pp. 302-307, 1966.

8. Y.H. Liu, Q. H. Liu and Z. P. Nie, “A New Efficient FDTD to Frequency DomainConversion ALghorithm," PIER progress in Electromagnetic Research, vol. 92, pp. 33-46, 2009.

9. D. Rakic, A. B. Djurisic, J. M. Elazar and M. L. Majewski, "Optical Properties of Metallic Films for Vertical Cavity,” Applied Optics, vol. 37, no. 22, pp. 5271-5283, 1998. 